\title{
A Breast Pump with a Compression Component is the Breast Pump of the Future
}

\author{
Alekseev NP ${ }^{*}$, Ilyin $\mathrm{VI}^{2}$ and Uleziko $\mathrm{VA}^{3}$ \\ ${ }^{1}$ Departments of General Physiology, Faculty of Biology, Saint Petersburg State University, Russia \\ ${ }^{2} \mathrm{OOO}$ Kapella, Russia \\ ${ }^{3} \mathrm{ZAO}$ PK Medthechnika, Russia
}

${ }^{\star}$ Corresponding author: Nikolay Alekseev, Departments of General Physiology, Faculty of Biology, Saint Petersburg State University, Russia

Received: October 27, 2021; Accepted: November 05, 2021; Published: November 19, 2021

\section{Introduction}

Breastfeeding, due to its nutritional and immunological aspects, is the best source of food for the newborn [1]. In situation where is not possible to breastfeed a baby, for example, where the mother has returned to work or is otherwise temporarily separated from her baby, it is necessary for her to express breast milk for storage period of separation using a breast pump. Also, if an infant is unable to effectively draw out the milk, for example, due to premature birth, illness, or underdeveloped nursing reflex, it may be necessary to express the milk from the mother's breast using a breast pump [2]. To achieve lactation success before a baby's suckling can ensure the effective extraction of milk, breast pumps must meet specific physiological and mechanical requirements. In particular, breast pumps:1) must effectively stimulate the mechanoreceptors in areola to promote maternal secretion and milkejection reflex, 2) must effectively remove milk from the breast, at the same time, pumping should not be painful or lead to damage to the nipples and areola 3) should remove high quality milk.

It should be noted here that milk excretion by the baby is studied in detail [1,3-5]. It is shown that the baby, creating a pulsating vacuum in the mouth, additionally performs mechanical compression of the areolar region of the breast with gums and tongue. However, during the operation of the vacuum breast pump, these mechanical effects on the nippleareolar region of the mammary gland are not used, and until recently there was no experimental data on the role of such compression in the process of milk excretion. Based on the assumption that the mechanical compression plays an important role in the milk excretion process, a breast pump has been developed that performs mechanical squeezing of the areolar region during milk excretion, along with exposure to pulsed vacuum (the device was called "Lactopuls"). Currently, a number of important advantages of the Lactopuls apparatus over modern vacuum breast pumps have been proved. These data are presented in this article.

\section{Materials and Methods}

Thirty lactating women 25-34 years old, 5-8 weeks lactation who volunteered to be included in this study were examined. Six of them were primiparous breastfeeding mothers and three were multiparous mothers. All infants were born in term. The informed consent of the women was obtained according to the Declaration of Helsinki.

\section{Breast Pump with Compression Stimuli}

Just like vacuum electric breast pumps, the breast pump "Lactopuls" consists of a control unit with a compressor and a removable funnel-shaped cup that is placed on the breast of a woman. Schematically, the removable funnel-shaped cup is shown in Figure 1A. It consists of conical and cylindrical part (3). However, unlike vacuum breast pumps, the cup is made an elastic material, such as silicone rubber. The cone part is placed on the breast (1). On two opposite external sides, rigid plates (4) are contact with the elastic

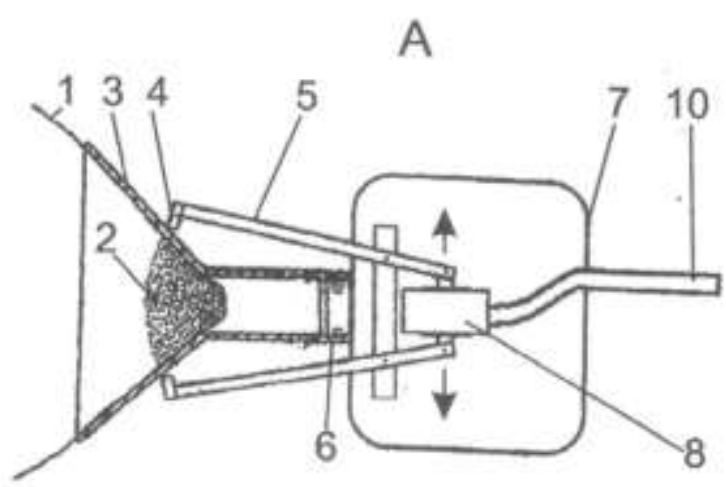

B

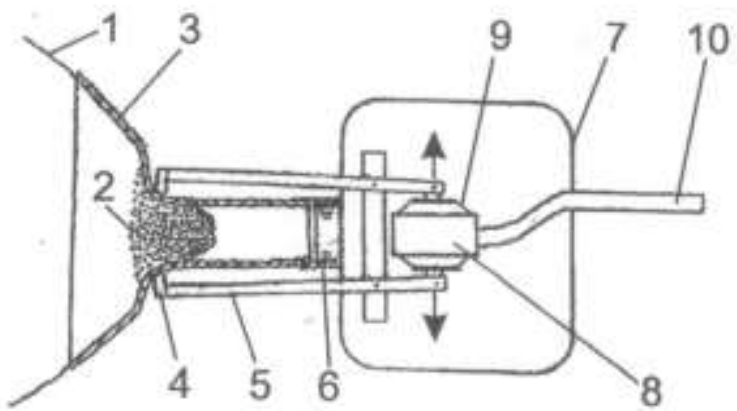

Figure 1: Action of the compression and vacuum component in the milk breast pump. A. Positive pressure pulse is not turned on. B. Positive pressure pulse is on.

1- mammary gland, 2- breast areola, 3-elastic cone, 4- rigid plates, 5- levers, 6- movable ledge, 7-solid body, 8- pneumatic pistons, 9- pneumatic pistons membranes, 10pneumatic hose 
cone, which are fixed at the front ends of the levers (5). The rear parts of the levers are connected to the movable membranes (9) of the pneumatic piston (8), to which excessive pressure pulse are supplied from control unit through the pneumatic hoses (10). The pneumatic piston is placed in the rigid body (7), to which a cylindrical part of the cup is attached on the outside on special movable ledge (6). The pump works as follows. Vacuum and compression (overpressure) stimuli are applied in certain sequence to the removable funnel-shaped cup from the control unit. Just as when milk is excreted by a child, at the beginning a vacuum acts on the mammary gland, which enters the cup via a pneumatic hose and the areola of the breast (2) begins to stretch, stimulating the skin stretching receptors. At the same time, under influence vacuum, milk begins to flow out of the ducts. After the same amount vacuum inside the elastic cone tube reaches the maximum value, positive pressure stimuli are applied to the piston. The movement of the piston membrane is transmitted to the levers (Figure 1B), and the rigid plates compress the conical part cup and areola, area where milk located. It is important to note here, that just as a child, the amplitude of compression and vacuum stimuli can independently regulate.

\section{Recording the Intraglandular Pressure}

In 11 women, the intraglandular pressure was recorded during expressing in the breast not being expressed. The breast was washed with anticeptic solution and one of the ducts was dilated with a special duct dilator. A metallic catheter with an outside diameter $0.5 \mathrm{~mm}$ was introduced into the duct as far as $2 \mathrm{~cm}$ approximately. The catheter was connected to the pressure gauge by means of polyethylene tube $0.7 \mathrm{~mm}$ in diameter. Before measuring, the whole system was filled with sterile saline $(0.9 \% \mathrm{NaCl})$ solution. The electric signal from the pressure gauge was conveyed to the amplifier and then to polygraph H-338 (Russia).

\section{Milk Analysis of Macronutrients}

Milk analysis of macronutrients (fat, proteins, carbohydrates) and energy value in the samples was performed 1-1.5 hours after milk ejection with a mid-infrared human analyzer Miris AB Uppsala, Sweden.

\section{Statistical Analysis}

Student s t-test was used for statistical analysis. In milk analysis experiments statistical analysis was performed using test two-way ANOVA. Large differences in nutrient concentrations in milk were found in women. Therefore, data for each woman were normalized relative to the concentration value of the first sample ejected from the breast by vacuum stimuli. Statistical significance was set as $\mathrm{p}<0.05$

\section{Results}

\section{Breast Pumps with Compression Component Effectively Stimulate the Mechanoreceptors in Areola to Promote Maternal Milk-ejection Reflex}

The normal functioning of the breast pump «Lactopuls» that is when vacuum and compression stimuli are applied to the nipple and areola resulted in milk flow in all the women. This could be clearly observed. Once the funnel attachment of the breast pump was applied to the breast milk began drip from the other breast after 0.5-1 min. Where the other breast was catheterized, an increase in intraglandular pressure occurred (Figure 2A). It should be noted that throughout the experiment visual observation revealed the pulsed character of milk removal in women. The periods of the absence of milk removal could be as long as 0.5-1 min. Yet graphically the pulsed character of milk removal was evident only when recording the milk volume with intervals of less than $1 \mathrm{~min}$ (Figure 2B). It was thought interesting to compare the pulsatory character of milk removal with intraglandular pressure. On comparing the graph Figure $2 \mathrm{~A}$ and $2 \mathrm{~B}$ it can be seen that the maximum milk removal rate coincides with the intraglandular pressure. Switching off the compression stimuli changed the dynamics of milk expression. The influence on milk removal of switching off the compression stimuli in women, who had difficulty expressing breast milk manually, was especially noticeable. The data from one woman presented in Figure 3 serves as an example. In the normal mode of the

$\boldsymbol{A}$
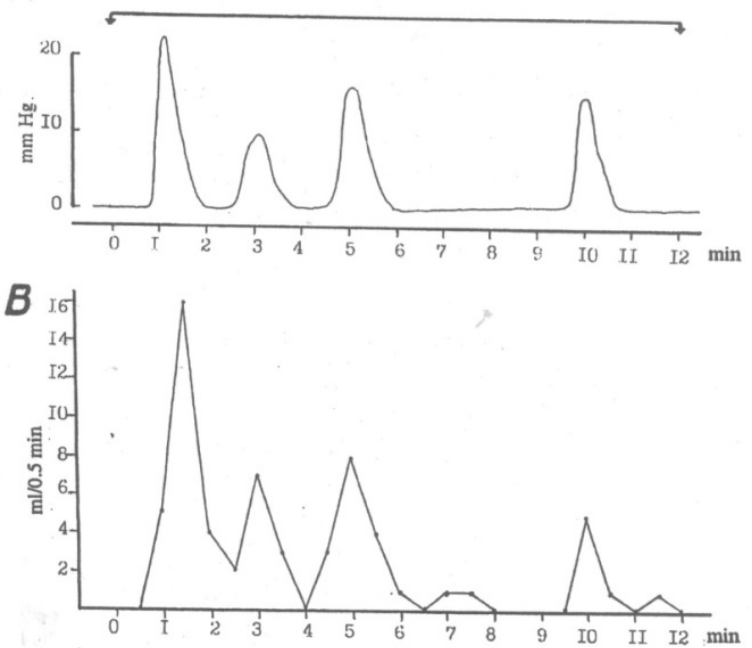

Figure 2: The change in intramammary pressure (A) and the rate of milk removal (B) Pressure in $\mathrm{mm} \mathrm{Hg}$, time in min. 

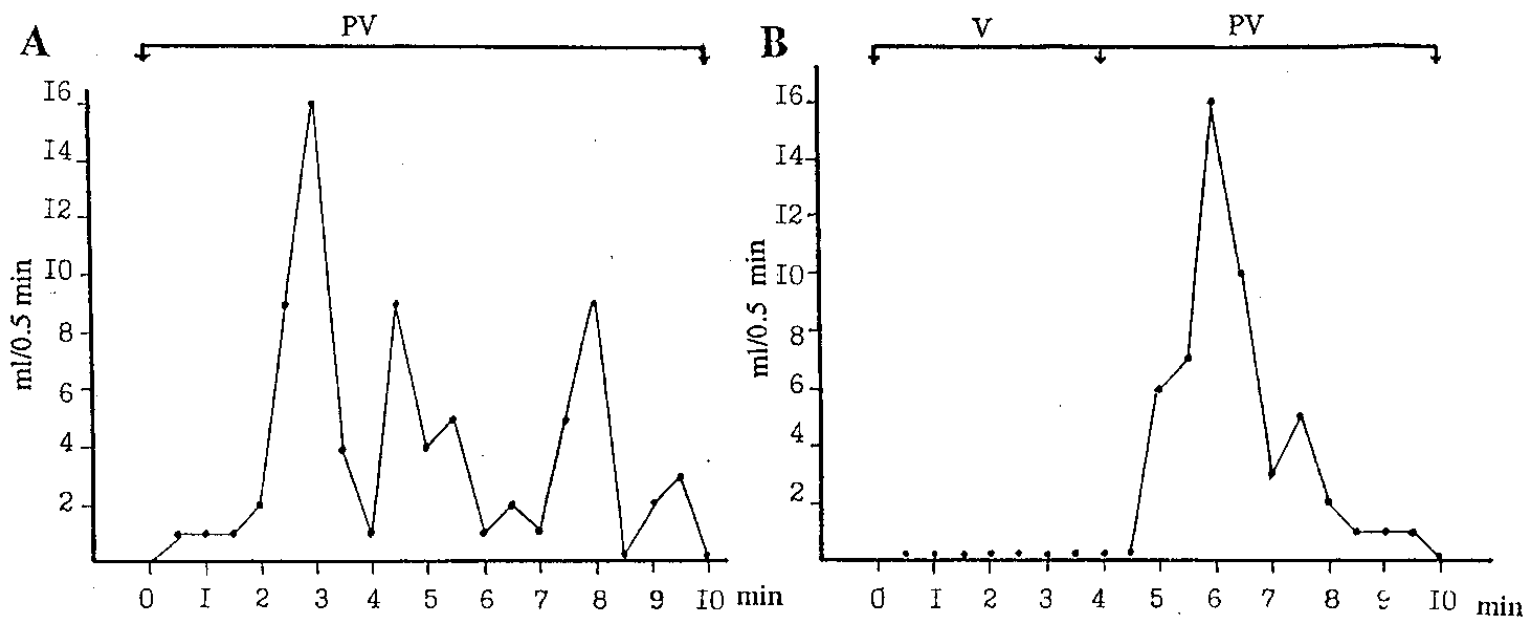

Figure 3: The change in volume of milk removed under different operating conditions of the breast pump. For A and B, the rate of milk removal ml/ $0.5 \mathrm{~min}$; time in min. In each graph the horizontal line shows the period of milk removal by means of the breast pump. PV, breast pump action in normal mode with both compression and vacuum stimuli; $\mathrm{V}$, breast pump action with compression stimuli switched off (vacuum only).

breast pump, that is when vacuum and compression stimuli are applied to the nipple and areola, the first peak of milk removal was reached in approximately $1.5 \mathrm{~min}$. During expression 6 peaks of milk flow were recorded in one woman (Figure $3 \mathrm{~A}$ ). When the compression stimuli were switched off milk did not appear for $4 \mathrm{~min}$ (Figure $3 \mathrm{~B}(\mathrm{~V})$ ). When compression was again switched on milk removal began in $0.5 \mathrm{~min}$ (Figure $3 \mathrm{~B}(\mathrm{PV})$ ), the maximum rate of the first peak reached $16 \mathrm{ml} /$ $0.5 \mathrm{~min}$, as it had been in the first case (Figure 3A).

Compression stimuli increase the efficacy of the breast pump which is not only due to the effective formation milk ejection reflex, but also directly to an additional squeezing the milk from the breast. At the same time, breast pump does not cause pain and does not damage the nipple and the areola of the breast.

Mechanical stimulation of mechanoreceptors of the areola releases oxytocin from the central nervous system into the bloodstream, which produces periodic increases in the intraductal mammary pressure. These serve to increase the rate of milk ejection (Figure 2). Evidently it is difficult to comparatively evaluate the effects of vacuum and vacuum-compression stimuli in milk ejection. We work out the method that allows us to reveal the difference in volume of milk expressed by applying purely vacuum and by applying vacuumcompression stimuli in relation to changes in intraductal pressure. This method will allow us to assess how the compressive force imposed on the mammary glands directly correlates with the volume of expressed milk.

According to the method, the nipple and areola were alternately affected by short (4s) series of $0.5 \mathrm{~s}$ vacuum stimuli with compression pulse of $0.27 \mathrm{~s}$. The amplitude of the vacuum pulses was within -120 and $-140 \mathrm{~mm} \mathrm{Hg}$, i.e. less than the maximum comfortable vacuum amplitude of $-191,3 \pm 6,5 \mathrm{~mm} \mathrm{Hg}$ [6]. Accordingly, the milk ejected to various milk collectors: (a) when pumping together with vacuum and compression stimuli and (b) when pumping only with vacuum stimuli (Figure 4A).

When pumping milk alternatively with vacuum pulses and vacuum with compression pulse, the volume of milk expressed together with
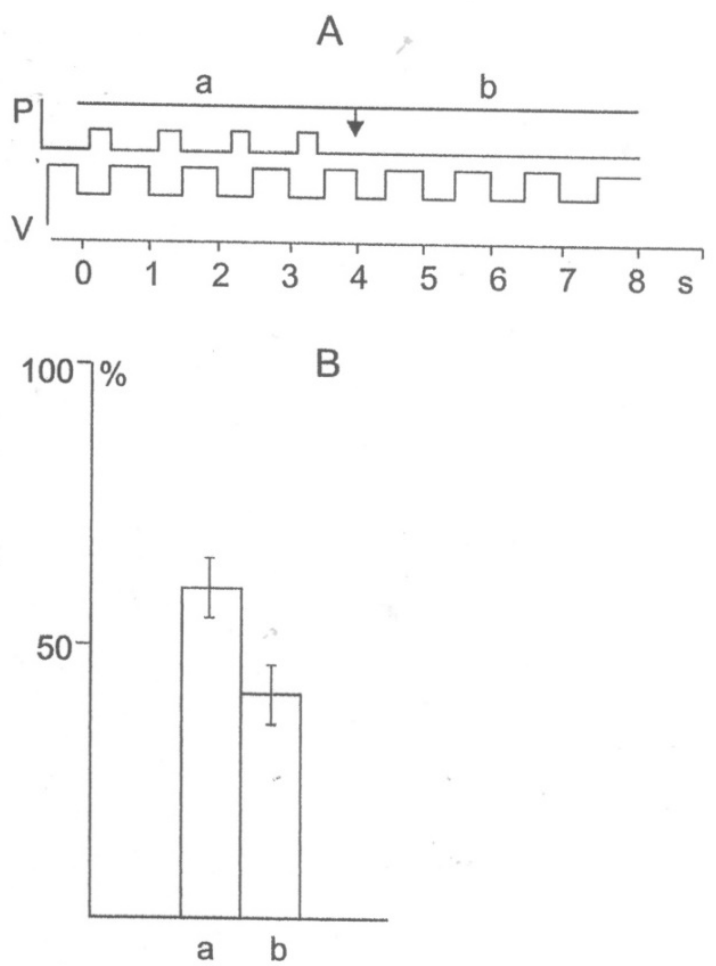

Figure 4: Influence of compression components on the amount milk removal.

A. Scheme of effects of vacuum and compressive stimuli on the mammary gland. (a) Simultaneous action of vacuum and compression stimuli, the milk enters the milk collectors (a); (b) action of vacuum stimuli, milk enters the milk collector (b). Ordinate, $\mathrm{P}$ (positive pressure), V (vacuum); abscissa, time (s). (B) The amount of milk as a percentage of the total volume of milk removal together by means of vacuum and compression pulses and by means of vacuum only (B). The ordinate: the amount of milk as percentage.

the help of vacuum and compression exceeded the amount of milk expressed single vacuum. However, the difference in volume varied in different patients in the range of $10 \%-46 \%$. On average , as follows from histograms (Figure 4B), the amount milk of expressed by one vacuum was $40.5 \pm 5 \%$, and the vacuum with compression stimuli was $59,5 \pm 5 \%$. 
In addition, according to Figure 5B, the volume of expressed milk equals40.5-1.03\% at vacuum milking and $59.5-1.07 \%$ at vacuumcompression milking. In this series of experiments, we additionally conducted comparative experiments on the expression milk of breast pump "Lactopuls" and one of the best vacuum breast pump Medela Symphony. It was found that the average values of the volume milk expressed with "Lactopuls" were 14\% higher, than volume of milk expressed with «Medela Symphony».

Milk ejection with vacuum and compression stimulus in breast pump increases amount of fat and protein in breast milk.

During observations among women, marked variations were found in the amount of milk ejected and in the concentration of milk nutrients studied. At the same time, the dynamics of nutrient concentrations in the process of milk ejection did not depend on the mode of operation of the apparatus and was similar for all women. Figure 5A shows graphs of changes in fat concentrations as well as the protein of milk (B) in $10 \mathrm{ml}$ samples during the ejection milk during the whole session of milk ejection. The largest changes in milk samples were observed in fat concentration (Figure 5A). The fat content in milk increased in each subsequent sample and in the latter samples exceed its content in the initial samples by 2-3,5 times. The total amount of ejected fat was about $25 \%$ more when milk ejected by vacuum with compression, than when milk ejected only by vacuum. When determining the protein concentration in milk samples, an increase in its concentration was also found during milk ejection, but on 10-20\% (Figure 5B). The increase in protein concentration occurred evenly during the entire ejection time. In contrast to the dynamics of fat and protein concentrations, the concentration of carbohydrates did not change in all women during of the milk ejection (Figure 5C).

The energy of milk is determined by the content of fat, protein, carbohydrates. It is easy to note that the graphs of changes in the energy value of milk (Figure 5D) have a great resemblance to the graphs of changes in the fat content of milk (Figure 5A).

\section{Discussion}

Presented experimental material shows that breast pump with compression component more effectively removes milk, and the quality of milk is also higher than milk extracted using vacuum. It can be assumed that one of the main reasons is the effective stimulation of the milk ejection reflex in a woman due to more adequate stimulation of the areola mechanoreceptors and, accordingly, an increase in oxytocin output [1]. An increase in the concentration of oxytocin will cause a more intensive contraction of the myoepithelial cells of the alveoli, which will "squeeze" more milk from the alveoli, including more caloric, i.e. with an increased fat and protein content, "hind milk". In addition to increasing the rate of milk excretion by increasing the pressure of milk in the ductal system of the gland (Figures 2 and 3) compression stimuli increase milk volume by compressing the expanded sections of the milk: "milk sinuses" located in the area of the areola mammary gland.

Observation during pumping indicate that the difference in the volume of expressed milk depend on anatomical characteristics of the breast. According to ultrasound studies, 6-14 milks ducts are suitable for the nipple, which at a distance of $8-9 \mathrm{~mm}$ from the base of the nipple
A

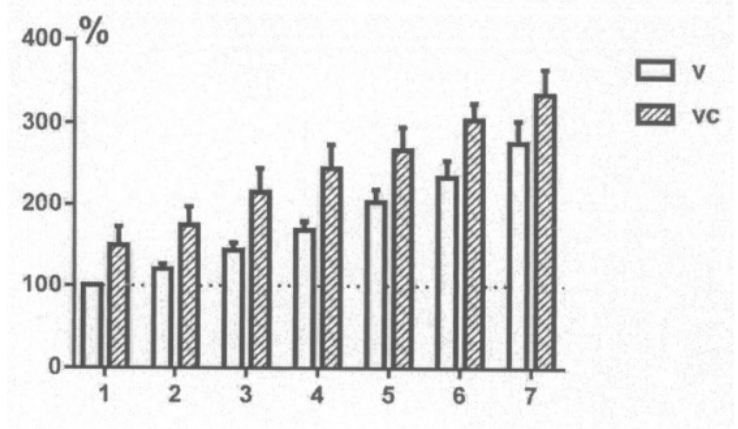

B

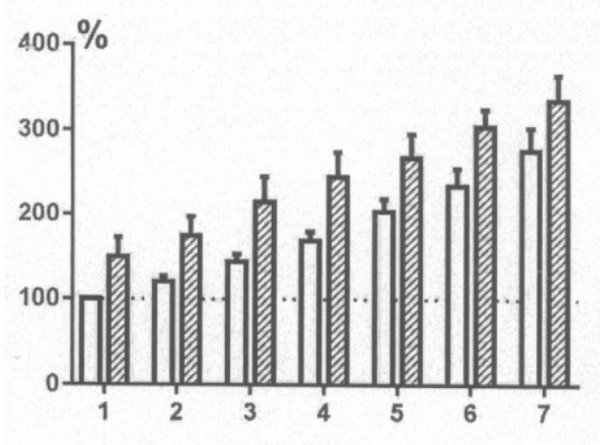

C

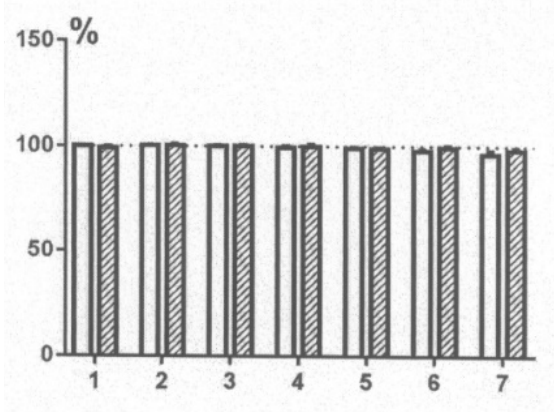

D

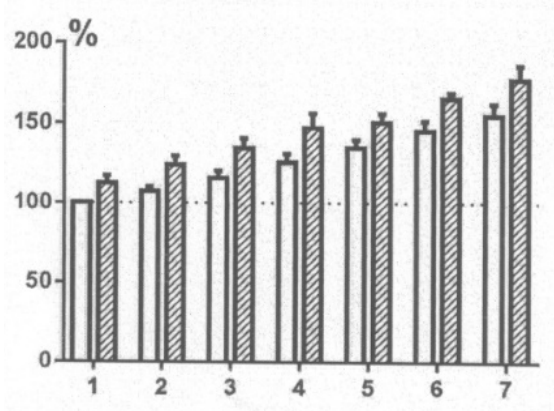

Figure 5: Change in the amount fat (A), protein (B), carbohydrates (C), and energy value (D) in woman in samples throughout the milk ejection session. 
have a maximum diameter of 1-5 mm ("milk sinuses") [7]. However, when the milk ducts pass into the milk ducts of the nipple, they narrow by five to ten times [8]. Moreover, the diameter of the milk ducts along the length of the nipple varies. So, when approaching the tip of the nipple ( $1 \mathrm{~mm}$ from the tip), the diameter of the ducts is the smallest and about $0.1 \mathrm{~mm}$. To the outlet, the ducts in most cases expand in the form of funnel with an increase in diameter by 1,5-3 times. When moving deep into the nipple (3.5-4mm from the tip) in different women, the diameter of the ducts increases and reaches $0.4-0.8 \mathrm{~mm}$. Then the diameter decreases again to an average of $0.4 \mathrm{~mm}[9,10]$. Since the resistance to the movement of liquid in the tube is inversely proportional to the area of its cross-section, nipples with thin milk ducts will inhibit the output of milk to a greater extent, and in this case, the mammary glands are classified as "tight". In addition, in the first 4 days after birth, when colostrum is present in the ductal system, the viscosity of which is higher than that of transitional and mature milk, difficulties with milk excretion are aggravated. To overcome the "tightness" of the gland, it is necessary to increase the pressure difference between the environment and the milk inside the milk ducts, for example, to increase the vacuum. However, as clinical studies have shown, high vacuum causes pain in the nipple, which inhibits the formation of the milk ejection reflex and consequently slows down the milk output. The child gets out of position by adding compression impulses to the vacuum stimuli so that the overall pressure difference between the environment and the milk inside the milk ducts increases markedly. In particular, when removing milk, the child can create a maximum vacuum of $197 \pm 10 \mathrm{~mm} \mathrm{Hg}$. The maximum amplitude of the compression pulses could reach $70 \mathrm{~mm}$ Hg. Thus, when added together, the total pressure difference will be about $270 \mathrm{~mm} \mathrm{Hg}$, which will significantly increase the efficiency of removing milk from the "tight" gland. At the same time, the woman will not experience discomfort, since when the child creates vacuum and compression stimuli, the values of vacuum and compression for the woman will be comfortable. The combination of vacuum and compression stimuli in the executive mechanism of the pump was also effective. Surveys have shown that compression stimuli against the background of vacuum stimuli $-120,-140 \mathrm{mmHg}$ can increase the output of milk from the gland by $46 \%$. Compression stimuli made a particularly noticeable contribution when pumping "tight" mammary glands and mammary glands in the first days of lactation filled with colostrum. Here it is interesting to note the results of surveys of Morton J [11]. In this work, against the background of sucking milk using a Medela Symphony breast pump, the fingers were additionally compressed in the area of the breast in front of the edge of the hard cup. As a result of the combination of vacuum and compression, the amount of milk produced increased significantly (up to $48 \%$ ). This method was especially effective when removing colostrum from the gland.

However, in the case of equal volume of milk expressed by vacuum and vacuum with compression pulses, examinations showed that the patient's breast was very "light". Through the transparent cover, it was clearly visible that the milk began to be released from the breast in trickles already under the influence of a vacuum of 60-70 mm Hg, i.e. half the established amount. Moreover, the milk as a result of the reflex of milk excretion began to drip quite intensively from the neighboring breast. In this case, the milk ducts in the nipple probably had a maximum diameter $(0.6-0.8 \mathrm{~mm})$ and a vacuum stimulus, before the compressing stimulus took effect, removed most of the milk. In this regard, it is interesting to note the results of a study of the process of removing milk from bottles, the nipples of which have holes for the exit of milk of different diameters (Eishima, 1991). It was discovered that the child had the greatest compressive effect tongue and gums on the nipples without holes or nipples with a very small diameter exit hole, which when you turn the bottle in vertical position water is dripping at a speed of $0.04 \mathrm{ml} / \mathrm{h}$. If the whole diameter was increased so that water dripped at a rate of $0.1 \mathrm{ml} / \mathrm{sec}$, the nipple compression was significantly weakened.

Here it should also be noted that when the milk was removed by a breast pump with a compression component, there was no compaction and puffiness of the areolar area of the breast. Massage of the areola with compressive stimuli as the milk is withdrawn, as well as in the case of milk withdrawal by a child, did not allow for compaction and puffiness of the areola. Therefore, the use of the breast pump in clinical practice with a compression component was effective in eliminating postpartum breast engorgement, as well as in the case of elimination of edema in lactostases. Thus, the use of milk-removing devices and especially devices with a compression component is an effective nondrug means of increasing the productivity of lactating women and increasing their lactation period.

\section{References}

1. Alekseev NP (2019) Physiology of human female lactation. Springer

2. Fewtrell MS, Lucas P, Collier S, Singhal A, Ahluwalia JS, et al. (2001) Randomized trial comparing the efficacy of a novel manual breast pump with a standard electric breast pump in mothers who delivered preterm infants. Pediatrics 107: 1291-1297. [crossref]

3. Ardran GM, Kemp FH, Lind J (1958) A cineradiographic study of breast feeding. $\mathrm{Br}$ J Radiol 31: 156-162. [crossref]

4. Mizuno K, Ueda A (2001) Development of sucking behavior in infants with Down's syndrome. Acta Paediatr 90: 1384-1388. [crossref]

5. Elad D, Kozlovsky P, Blum O, Laine AF, Po MJ, et al. (2014) Biomechanics of milk extraction during breast-feeding. Proc Natl Acad Sci USA 111: 5230-5235. [crossref]

6. Mitoulas LR, Lai CT, Gurrin LC, Larsson M, Hartmann PE (2002) Effect of vacuum profile on breast milk expression using an electric breast pump. J Hum Lact 18: 353360. [crossref]

7. Prime DK, Geddes DT, Spatz DL, Robert M, Trengove NJ, et al. (2009) Using milk flow rate to investigate milk ejection in the left and right breasts during simultaneous breast expression in women. Int Breastfeed J 4: 1-10. [crossref]

8. Ramsay DT, Kent JC, Hartmann RA, Hartmann PE (2005) Anatomy of the lactating human breast redefined with ultrasound imaging. J Anat 206: 525-534. [crossref]

9. Going JJ, Mohun TJ (2006) Human breast duct anatomy, the 'sick lobe' hypothesis and intraductal approaches to breast cancer. Breast Cancer Res Treat 97: 285-289. [crossref]

10. Rusby JE, Brachtel EF, Michaelson JS, Koerner FC, Smith BL (2007). Breast duct anatomy in the human nipple: three-dimensional patterns and clinical implications. Breast Cancer Res Treat 106: 171-179. [crossref]

11. Morton J, Hall JY, Wong RJ, Thairu L, Benitz WE, et al. (2009) Combining hand techniques with electric pumping increases milk production in mothers of preterm infants. J Perinatol 29: 757-764. [crossref]

\section{Citation:}

Alekseev NP, Ilyin VI, Uleziko VA (2021) A Breast Pump with a Compression Component is the Breast Pump of the Future. Integr Gyn Obstet J Volume 4(4): $1-5$. 